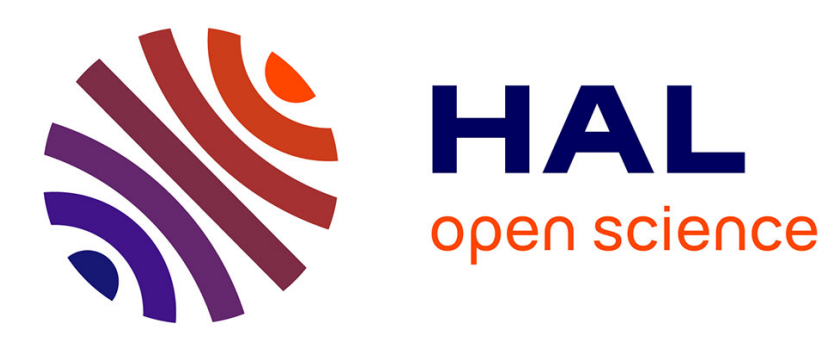

\title{
La série céramique du Bronze ancien 1 au Roc d'en Gabit, Carcassonne (Aude)
}

Jean Vaquer, Maxime Remicourt

\section{To cite this version:}

Jean Vaquer, Maxime Remicourt. La série céramique du Bronze ancien 1 au Roc d'en Gabit, Carcassonne (Aude). Bulletin de la Société préhistorique française, 2008. hal-02050718

\section{HAL Id: hal-02050718 \\ https://hal-univ-tlse2.archives-ouvertes.fr/hal-02050718}

Submitted on 1 Mar 2019

HAL is a multi-disciplinary open access archive for the deposit and dissemination of scientific research documents, whether they are published or not. The documents may come from teaching and research institutions in France or abroad, or from public or private research centers.
L'archive ouverte pluridisciplinaire $\mathbf{H A L}$, est destinée au dépôt et à la diffusion de documents scientifiques de niveau recherche, publiés ou non, émanant des établissements d'enseignement et de recherche français ou étrangers, des laboratoires publics ou privés. 


\begin{abstract}
Résumé
Le site de Roc d'en Gabit à Carcassonne est un établissement de plein air fortifié par une enceinte à fossé qui a livré un abondant mobilier du début de l'Âge du bronze ancien. Hormis quelques éléments plus anciens, en position secondaire dans le comblement des structures en creux, la céramique de ce site paraît très homogène. Elle provient essentiellement de rejets domestiques et elle est représentative de l'étape la plus ancienne du Bronze ancien méridional. La série analysée sous ses aspects typologiques comporte trois sortes de céramiques : des céramiques fines décorées dans le style épicampaniforme barbelé, des céramiques fines non décorées comportant des tasses et gobelets à anse et de la céramique épaisse de stockage et culinaire à fond plat de style rhodanien. Les comparaisons ont été effectuées avec les séries de plusieurs sites du Midi, dans deux tableaux d'occurrences des formes et des décors d'ensembles campaniformes des styles régionaux et d'ensembles épicampaniformes du début de l'Âge du bronze ancien. Le traitement par indice de similarité de Jaccard indique une forte parenté de la série de Roc d'en Gabit avec celles du style de Camp de Laure, notamment pour les décors, et une dérivation probable de la céramique commune des groupes pyrénéen et rhodano-provençal pour les formes. Les liens avec le Nord-Est de l'Espagne sont beaucoup plus réduits.
\end{abstract}

\begin{abstract}
The site of Roc d'en Gabit in Carcassonne is an open-air settlement fortified by a ditch enclosure that has yielded numerous artefacts from the beginning of the Early Bronze Age. Apart from a few older elements, in a secondary position in the filling of hollow structures, the ceramics from this site seem to be very homogeneous. They come mainly from household waste and are representative of the earliest stage of the southern Early Bronze Age. The series analysed by typological aspects included three kinds of pottery: fine ceramics decorated in the «barbelé» «épicampaniforme» style, undecorated fine ceramics including cups and handled beakers, and the thick storage and flat-bottomed utility ware of the "Rhodanian" style. Comparisons have been made with series from several sites in southern France, in two occurrence tables of the shapes and decorations of Bell Beaker assemblages for regional styles and for «épicampaniforme» assemblages of the beginning of the Early Bronze Age. Treatment with the Jaccard similarity index indicates a strong similarity between the series of Roc d'en Gabit and the style of Camp de Laure especially for the decorations, and a likely derivation of common ware from Pyrenean and Rhodano-Provençal groups for the shapes. Links with the north-east of Spain are much less marked.
\end{abstract}


Le Bronze ancien du Languedoc méditerranéen occidental a d'abord été perçu à partir des objets métalliques et rattaché à la civilisation rhodanienne (Bailloud, 1966). L'existence de survivances campaniformes au début du Bronze ancien a été reconnue par J. Guilaine en 1967 sous forme d'une étape épicampaniforme à céramique décorée au stylet ou en lignes «rouletées». Ce style obtenu surtout par impression d'une tige filetée a été nommé «barbelé» par J. Bill (1973, 1976 et 1977). Il a été défini stylistiquement par J. Courtin à partir des séries conséquentes du Camp de Laure au Rove dans les Bouches-du-Rhône, un établissement fortifié qui est devenu le site éponyme d'un style céramique du Bronze ancien I méridional, présentant un syncrétisme d'aspects campaniformes, italiques et rhodaniens (Courtin, 1975). La reconnaissance des céramiques communes, non décorées à fond plat, des faciès campaniformes maritime, pyrénéen et rhodano-provençal, issues de la composante «RhinRhône» (Gallay, 1986), a impliqué de les prendre en compte comme un legs campaniforme supplémentaire dans le style du Camp de Laure (Guilaine et al., 1989; Besse, 1996). L'horizon à décor barbelé a été ensuite reconnu sous divers aspects dans l'ensemble du Midi comme une composante de plusieurs faciès locaux à céramique de tradition régionale ou en relation avec la culture du Rhône (Guilaine, 1996). D'autres auteurs ont considéré qu'il s'agissait d'un horizon de filiation campaniforme sortant de la stricte définition socioéconomique du Bronze ancien (Strahm, 1998). Plus généralement, selon les modèles du colloque de Riva del Garda (Guilaine et al., 2002), il est proposé de voir le Campaniforme ancien ( $A O C$ et maritime) comme greffé aux cultures chalcolithiques locales, puis de considérer qu'il y a une autonomisation culturelle au moment du Campaniforme épimaritime. Les groupes campaniformes pyrénéen et rhodano-provençal sont considérés comme transgressifs sur les cultures chalcolithiques locales en voie d'acculturation. L'horizon barbelé se développerait partout dans le Midi après l'extinction des cultures chalcolithiques locales à partir d'un fonds campaniforme et avec intégration de nouvelles influences orientales (Italie-Europe centrale) sensibles au moins jusqu'aux Pyrénées (Lemercier, 2004). La question d'une éventuelle survivance de caractères chalcolithiques indigènes dans les séries du Bronze ancien I se pose pour les zones périphériques ou peu marquées par l'impact campaniforme, comme c'est le cas dans la bordure méridionale du Massif central, notamment pour le groupe des Treilles récent, ou dans le Sud-Ouest pour l'Artenac final (Hénaff, 2003). Dans le piémont nord-pyrénéen, la situation est moins claire, les ensembles disponibles sont souvent peu étoffés et ne présentent que rarement des conditions garantissant une réelle homogénéité car les séries proviennent de grottes ou de récoltes de surface après défonçage de vignes. Dans le Toulousain, quelques assemblages ont été constitués à partir de nappes de vestiges fouillées lors d'opérations préventives sur les sites de Lapeyrère à Muret (Jolibert, 1988) et plus récemment sur les sites contigus de Cassagna 2 et 3 à Blagnac en Haute-Garonne (Pons et al., 2003;
Tchérémissinoff et al., 2005). Ils ont donné lieu à des interprétations différentes selon la chronologie et la durée envisagée pour leur constitution. La fouille récente d'une enceinte du début du Bronze ancien sur le site de Roc d'en Gabit à Carcassonne (Aude) vient de livrer une importante série de céramiques qui se place à la jonction des aires relevant du style barbelé méditerranéen et des groupes périphériques à survivances chalcolithiques indigènes supposées. Il nous paraît donc intéressant d'en examiner la composition pour une réflexion générale sur les traditions potières dans ce cadre de transition à la fois temporel et géographique.

\section{LE SITE DE ROC D'EN GABIT, ENCEINTE ET STRUCTURES DU BRONZE ANCIEN}

\author{
Historique \\ de la découverte
}

Le gisement du Roc d'en Gabit, au nord de la commune de Carcassonne près du hameau de Montredon, est situé sur un lambeau de terrasse rissienne d'une dizaine d'hectares dominant l'Aude. L'ossature de cette colline est constituée par un banc de grès issu de la formation des molasses lutétiennes de Carcassonne. Dans la partie est du plateau, c'est une nappe graveleuse qui affleure, à l'ouest une formation lœssique provenant des dépôts éoliens du Pléniglaciaire wurmien. C'est cette zone qui a livré les vestiges archéologiques. Les documents cadastraux anciens indiquent que c'est juste en face de cette petite butte que le Fresquel confluait avec l'Aude, avant la construction du canal du Midi. Détecté il y a plus d'une trentaine d'années par J. Vaquer, lors d'une série d'arrachage de vignes, le gisement a livré des structures archéologiques délimitant des zones d'occupation qui s'échelonnent du Néolithique moyen chasséen jusqu'à l'époque médiévale. Cette longue durée des réoccupations successives est sans doute à mettre en relation avec la position géographique à la fois stratégique et économique que représente la confluence des deux principales voies fluviales du couloir de l'Aude.

À la suite de défonçages en 1991, différents indices pédologiques sont apparus avec netteté et ont révélé par des photographies aériennes les tronçons d'un fossé annulaire, ainsi que des lambeaux de sol anthropisé. L'année suivante, une série de sondages mécaniques a permis de montrer que le diamètre de l'enceinte annulaire était de l'ordre de $100 \mathrm{~m}$. Le fossé, dans les zones les moins érodées, mesurait entre 8 et $10 \mathrm{~m}$ de large, avec une section en $U$ pouvant atteindre $3 \mathrm{~m}$ de profondeur. Le matériel archéologique était attribuable au Néolithique final vérazien, en accord avec la datation ${ }^{14} \mathrm{C}$ de la couche 4 : Ly $6294: 4380 \pm 95$ BP, soit 33302784 av. J.-C. (Vaquer, 1999; Vaquer et al., 2004). En 1999, l'implantation d'une carrière pour l'exploitation du grès a détruit une partie du gisement dans la zone où se situait l'occupation campaniforme. Pour faire face à l'extension de la carrière et aux menaces de 
destructions par des emprunts de terre et des travaux agricoles, une importante opération de fouilles programmées a été conduite de 2000 à 2004, qui a permis une exploration globale du site. Outre les occupations chasséennes, véraziennes et campaniformes, ces travaux ont permis de mettre en relief une enceinte de l’Âge du bronze ancien.

\section{L'enceinte et les structures du Bronze ancien}

Lors de la campagne de fouilles de 2000, un fossé très arasé a pu être reconnu. Il recoupait la grande enceinte annulaire du Néolithique final en deux points. Il semble que celle-ci ne devait plus être visible à l'époque (fig. 1). Ce fossé se compose de trois tronçons qui ont été presque intégralement fouillés.

Le tronçon F24 au nord-ouest est attesté sur $31 \mathrm{~m}$ de long, avec une orientation nord-sud. Il est tronqué par l'érosion vers le sud et se dirige vers la falaise de grès au nord. Il a une section en U et mesure $4 \mathrm{~m}$ de large, sa profondeur maximale conservée est de 1,30 m, mais à aucun endroit il n'a pu être raccordé un sol de circulation. Ce tronçon est nettement en pente vers le nord puisque la base passe d'une altitude de $107,10 \mathrm{~m}$ à l'extrémité sud à 104,02 m dans la coupe nord du sondage 2 , soit une pente de $10 \%$.
Le tronçon F18 dans la parcelle 49 est pratiquement rectiligne et orienté est-ouest, et se développe sur $39 \mathrm{~m}$. Il était tronqué par l'érosion vers l'ouest et par un emprunt de terre vers l'est. Dans ce secteur, seule la base du fossé est conservée; elle a une section en $U$ avec des parois verticales et forme parfois des sortes de compartiments séparés par des paliers. Il mesure au mieux $3,80 \mathrm{~m}$ de large et $0,70 \mathrm{~m}$ de profondeur. Sa base est fortement en pente vers l'est puisque l'altitude de la base est de $106 \mathrm{~m}$ à l'extrémité ouest et de $103 \mathrm{~m}$ à l'extrémité est, soit une pente de $8 \%$.

Le dernier tronçon F43 se trouve dans le prolongement du tronçon F18; il a pu être dégagé sur 16 m de long. Il est nettement incurvé et légèrement élargi au niveau du recoupement avec le fossé néolithique et s'interrompt brutalement sur le point le plus élevé de son développement à l'ouest. L'ensemble forme une sorte d'antenne près d'une interruption probablement située dans l'angle sud-ouest de l'enceinte. Le fossé devait se prolonger ensuite vers le nord pour rejoindre le tronçon F24. Les diverses coupes relevées montrent que le tronçon F43 a un pendage vers l'est, sa base se trouvant à la cote $107,40 \mathrm{~m}$ à l'extrémité ouest et à $106,90 \mathrm{~m}$ dans la coupe la plus à l'est. Il s'agit évidemment d'une structure très arasée qui est conservée sur 0,75 à $0,55 \mathrm{~m}$ de profondeur. Comme dans le secteur F18, la section est trapézoïdale ou en $\mathrm{U}$, avec un fond

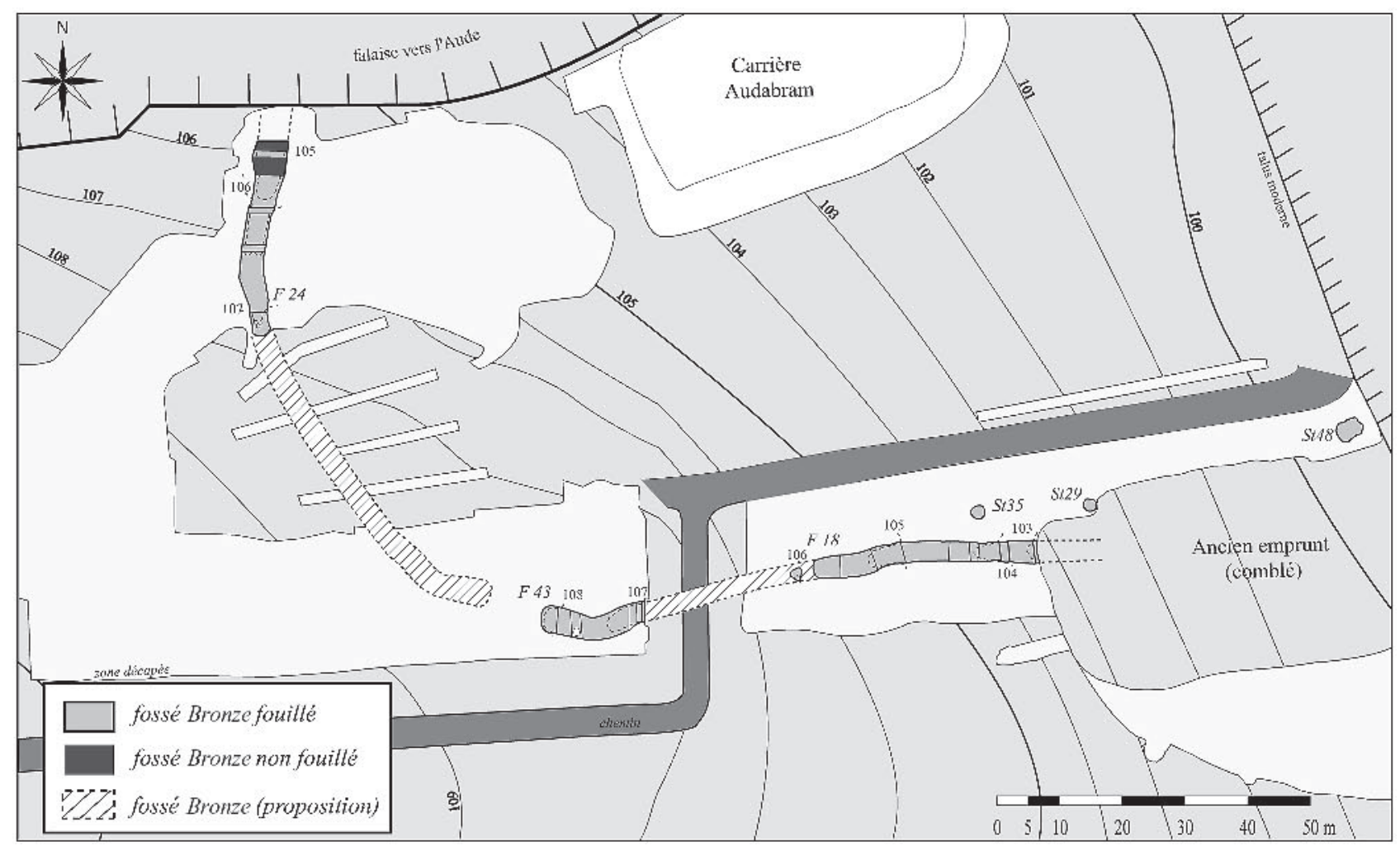

Fig. 1 - Roc d'en Gabit, Carcassonne (Aude). Plan des structures de l'Âge du bronze ancien. F 18, F 24 et F 43 correspondent aux tronçons conservés du fossé d'enceinte dont le tracé restitué est proposé en hachuré. Les structures St 35 et St 29 correspondent à des bases de silos et la structure St 48 à une fosse d'extraction (relevé J. Vaquer, DAO H. Vergély et M. Remicourt).

Fig. 1 - Roc d'en Gabit, Carcassonne (Aude). Plan of the Early Bronze Age structures. F 18, F 24 and F 43 correspond to the preserved sections of the ditch enclosure for which the proposed layout is shown in hatching. The structures St 35 and St 29 correspond to the foundations of silos and structure St 48 to an extraction pit (drawing J. Vaquer, CAD H. Vergély and M. Remicourt). 
plat ou légèrement concave de 3 à $4 \mathrm{~m}$ de large et avec des parois droites ou fortement inclinées, voire subverticales.

Tout au long de son développement, ce fossé a reçu les mêmes types de dépôts de comblement. À la base, le remplissage ne révèle aucune asymétrie des apports qui pourraient indiquer l'existence d'un talus ayant bordé le fossé. Les coupes transversales montrent trois épisodes de comblement qui semblent obéir à une dynamique privilégiant les apports latéraux au début, puis des rejets anthropiques dans un second temps et enfin une dynamique de transit longitudinal de sédiments issus de l'érosion.

L'épisode 1 est surtout représenté par des apports localisés sur les côtés qui correspondent probablement à l'effondrement ou à l'érosion des parois du fossé. Il s'agit de lœss remanié lorsque le fossé est creusé dans le lœss ou de limon brun dans le secteur du recoupement avec le fossé néolithique, ce qui indique que ces dépôts ont été rapides et peu déplacés.

L'épisode 2 est marqué par des apports de limon brun mêlés à des résidus de combustion, des pierres chauffées et du mobilier relativement abondant, qui correspond assurément à la phase de fréquentation de l'enceinte. Localement des traces de feu in situ ont été mises en évidence dans les trois tronçons (zones de terre rubéfiée).

L'épisode 3 est représenté par des apports limoneux bruns, moins chargés en détritus et contenant de nombreux restes de gastéropodes. Il correspond surtout à des apports liés au ruissellement et à l'érosion du paléosol brun qui devait exister sur le site à cette époque.

Il semble acquis, malgré les lacunes dues à l'érosion, que ce fossé se développait sur le site en adoptant soit une morphologie en L et, dans ce cas, en prenant appui sur deux talus naturels au nord et à l'est, soit plutôt une forme en $U$ accolée à la falaise qui domine l'Aude. En effet, le talus actuel à l'est qui sépare les parcelles 50 et 53 est essentiellement artificiel et il est probable que le fossé devait revenir vers le nord audelà de ce talus où il est complètement détruit par des travaux de nivellement récents. Quoi qu'il en soit, ce fossé formait un barrage qui délimite un espace quadrangulaire bordé par l'escarpement de la terrasse sur le côté nord et dominant l'Aude d'une quinzaine de mètres. Compte tenu de l'érosion et des destructions récentes liées à des carrières, il est impossible de savoir si ce fossé était continu ou segmenté. Toutefois, le coude amorcé et l'interruption brutale visible au niveau du tronçon F43 suggèrent l'existence d'un passage à ce niveau. Les trois tronçons fouillés ont un pendage conforme à la topographie générale du site et même plus accentué que la pente naturelle du terrain. Ils ont probablement permis un drainage des eaux de pluie à la fois vers le nord et vers l'est. Le développement total de cette structure peut être estimé au minimum à $270 \mathrm{~m}$. Elle délimitait une superficie de 1,5 hectare, ce qui la place au même rang que l'enceinte du Camp de Laure au Rove dans les Bouches-du-Rhône (Courtin, 1975).

Les structures ponctuelles du Bronze ancien qui peuvent être contemporaines de l'enceinte se trouvent toutes à l'intérieur de celle-ci. Il s'agit de bases de grands silos pour les structures St 35 et St 29 et d'une fosse d'extraction pour la structure St 48 . Il faut noter que les fosses St 35 et St 29 ne sont pas éloignées du fossé $(3,30 \mathrm{~m}$ pour la structure 35 et sans doute la même chose pour la structure 29), ce qui laisse peu de place pour une simple levée de terre interne. La structure en élévation qui devait doubler le fossé était donc peut-être beaucoup plus élaborée et éventuellement impliquée dans l'architecture des habitats. Des fragments de grosses pièces en bois carbonisées qui ont été trouvées dans plusieurs secteurs du fossé (F24 et F43) suggèrent l'existence probable d'une palissade incendiée. Dans la mesure où aucune trace de ravinement n'a été observée dans les diverses coupes transversales du secteur F24 qui était déclive, il est possible d'envisager que le fossé ait été interrompu avant le rebord de la falaise. Cette interruption probable aurait fait barrage et favorisé une importante et rapide accumulation de sédiment.

\section{CARACTÉRISATION TYPOLOGIQUE DE LA SÉRIE CÉRAMIQUE DU ROC D'EN GABIT}

La série céramique la plus importante provient des divers tronçons du fossé d'enceinte. Dans le secteur sud (tronçons F18 et F43), cette céramique morcelée était essentiellement présente à la base du remplissage du fossé, qui était constitué de terre très cendreuse que nous interprétons comme des rejets de vidange de foyers et de détritus de consommation, malgré la présence de restes humains épars. Dans le secteur occidental de l'enceinte (secteur F24), le remplissage limoneux du fossé était beaucoup plus dilaté et la céramique distribuée sur l'ensemble du comblement.

Dans ces conditions, il apparaît comme très probable que la série céramique du fossé de l'enceinte du Bronze ancien de Roc d'en Gabit ait été constituée au cours d'une période relativement brève. La plupart des unités stratigraphiques qui ont livré le matériel correspondent à la base d'un fossé établi en terrain limono-lœssique extrêmement sensible à l'érosion. Les quelques raccords de tessons obtenus dans le secteur méridional de l'enceinte tendent à renforcer ce point de vue. Ils révèlent des liaisons entre des tessons situés à des niveaux différents qui couvrent la majeure partie de la séquence conservée.

Une datation a été obtenue pour cet ensemble à partir d'un gros charbon de la couche 3 du sondage 1 réalisé sur le tronçon F24. Le résultat de l'analyse radiométrique Ly 12022 : $3835 \pm 35$, soit $2455-2149$ av. J.-C. avec un maximum de probabilité à 23502150, paraît un peu haute par rapport aux séries de dates radiocarbone disponibles pour les ensembles à céramique barbelée du Midi, qui se placent plutôt entre 2100 et 1900 (Voruz, 1996; Vital, 2004). Cela pourrait être dû à un effet de vieux bois dans le cas de Roc d'en Gabit, qui n'est pas très éloigné de cette fourchette. 


\section{Éléments hérités des occupations antérieures}

Le fossé étant creusé sur un site occupé à diverses reprises au cours du Néolithique (Chasséen ancien et récent, Néolithique final saint-ponien) et du Chalcolithique (Vérazien et Campaniforme), il ne faut pas s'étonner de trouver dans le remplissage des éléments plus anciens en position secondaire (Vaquer et al., 2004). Ils ont pu parvenir au sein du remplissage soit par l'érosion aréolaire des paléosols contigus au fossé, soit par recoupement de structures antérieures. Cet état de fait est attesté pour le fossé annulaire du Néolithique final recoupé dans le secteur F43 et dans le secteur F24. C'est le cas aussi pour un silo vérazien tronqué dans le secteur F18.

Les plus anciens vestiges, en position secondaire, sont des tessons de style chasséen, notamment des bords de coupes à sillon interne sous la lèvre, des fragments d'écuelles à micro-épaulement ou des fragments de mamelons perforés.

On peut mentionner également quelques éléments lithiques. Une perle discoïde en stéatite appartient à un type généralement considéré comme datant du Néolithique final ou du Chalcolithique. Un galet en calcaire blanc à cupules opposées correspond à une ébauche de bracelet d'un type très fréquent dans l'enceinte annulaire du Néolithique final. On peut mentionner aussi un fragment de bracelet à section rectangulaire à trois gorges qui correspond au même type qu'un autre fragment trouvé dans une structure campaniforme du gisement.

La série céramique n'est pas exempte de telles intrusions d'éléments plus anciens. On peut mentionner notamment des fragments de vases à fond rond, tels que des gobelets à profil galbé, des jattes hémisphériques ou des bols tulipiformes pourvus de mamelons ronds qui évoquent les productions céramiques véraziennes. La même attribution est possible pour quelques fragments de grands vases munis de languettes de préhension sous le bord.

Deux tessons décorés typiques du complexe campaniforme ont été identifiés dans la série du fossé. Il s'agit d'un bord de gobelet de style maritime qui présente sous la lèvre interne des lignes d'impressions à la cordelette et un décor externe de bandes horizontales, délimitées à la cordelette, avec un remplissage de hachures obliques au peigne (type Ilvesheim). L'autre tesson est un fragment de gros vase de style épimaritime, probablement une jatte à profil galbé dont la panse porte trois bandes hachurées au peigne et de sens alterné.

Toutefois, ces éléments hérités ne composent qu'une infime partie du mobilier retrouvé dans le comblement du fossé.

\section{La céramique du Bronze ancien}

La céramique trouvée dans le fossé est abondante et relativement bien conservée, ce qui a permis la restitution graphique complète ou partielle d'une trentaine de vase. Ce lot, qui est un des plus représentatifs actuellement connu dans le bassin de l'Aude, peut être scindé en trois sortes de productions.

\section{La céramique fine décorée dans le style épicampaniforme}

Cette céramique de haute qualité à dégraissant très fin est représentée par une trentaine d'éléments. Les indicateurs morphologiques permettent de reconnaître des formes de tradition campaniforme comme un gobelet bas à profil galbé (fig. $2, \mathrm{n}^{\circ} 2$ ) et un vase bitronconique large à bord relevé (fig. $2, \mathrm{n}^{\circ} 6$ ). Un grand vase bitronconique élancé à col concave est plus original (fig. 2, $\mathrm{n}^{\circ}$ 9), quoique des formes proches soient connues dans la céramique lisse du groupe rhodanoprovençal, par exemple aux Calades d'Orgon, Bouchesdu-Rhône (Barge-Mahieu, 1992). La plupart des tessons décorés appartient à des tasses carénées hautes ou à des gobelets bitronconiques portant une décoration qui peut être limitée à l'espace au dessus de la carène (fig. $2, n^{\circ} 7$ ) ou bien couvrir aussi la partie inférieure de la panse (fig. $2, \mathrm{n}^{\text {os }} 1$ et 6 ). Ce sont ces vases qui devaient être pourvus des quelques anses décorées figurant dans la série. Ces anses en ruban joignent la carène et le bord, selon une disposition qui est typique du Bronze ancien de style Camp de Laure.

Les décors ont été réalisés avec plusieurs techniques sur pâte encore plastique. On reconnaît des impressions courtes réalisées avec une petite pointe de façon à obtenir des trous ovales ou des traits courts. L'emploi d'une tige pleine a donné des ponctuations rondes et l'emploi d'une tige creuse des impressions circulaires «au chalumeau» qui sont les plus typiques de ce style. D'autres impressions ont été réalisées avec des matrices dentées, probablement des peignes, de façon à obtenir de fines lignes de pointillés (fig. $2, \mathrm{n}^{\text {os }} 5$ et 8 ), voire des lignes barbelées à la tige souple filetée (fig. 2, nº 4). Les incisions ont été réalisées avec un outil tranchant ou mousse donnant soit de fines lignes, soit des sillons (fig. $2, \mathrm{n}^{\circ} 1$ ). Les combinaisons de techniques pour un même effet décoratif sont attestées notamment pour les incisions recoupées par des traits au poinçon donnant le classique motif incisé barbelé ou incisé frangé tel qu'il est observé sur le seul gobelet restituable (fig. 2, $n^{\circ} 2$ ). Le décor très finement cranté à la tige souple filetée, serrée, ne semble attesté que dans quelques cas, qui ne sont pas très sûrs étant donné la mauvaise conservation des tessons. Cette technique utilisée surtout pour fixer une incrustation blanche est attestée dans l'Aude à la grotte des Chambres d'Alaric à Moux et à l'abri de Font-Juvénal à Conques-sur-Orbiel. Il ne serait pas étonnant de l'observer à Roc d'en Gabit.

Les motifs décoratifs sont essentiellement des bandes dont la disposition est plus souvent horizontale (fig. 2). On connaît aussi des bandes verticales, c'est le cas notamment des anses décorées mais aussi des décors qui ornent la partie inférieure de quelques tasses hautes ou gobelets bitronconiques en formant des motifs rayonnant vers le fond, selon une modalité bien 

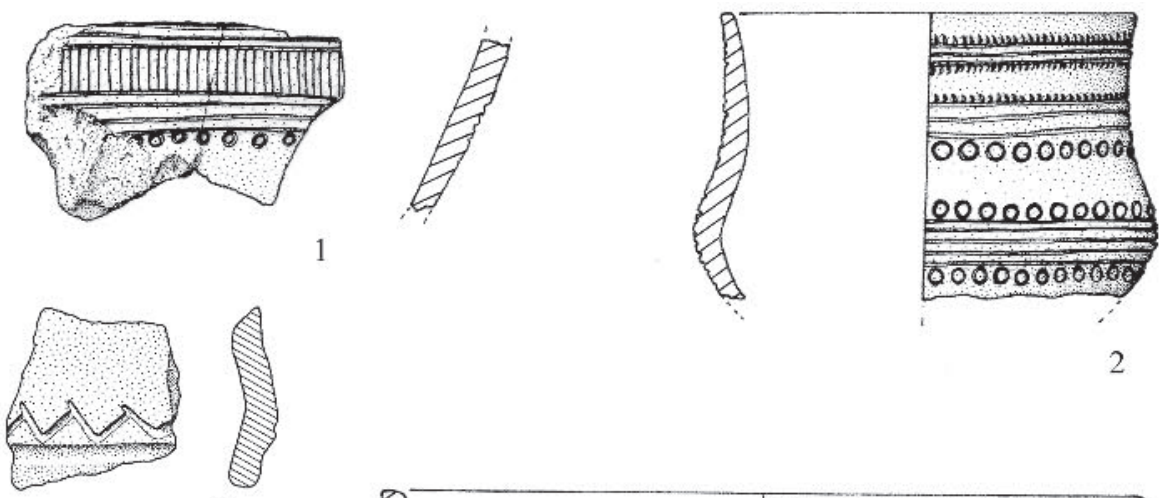

2
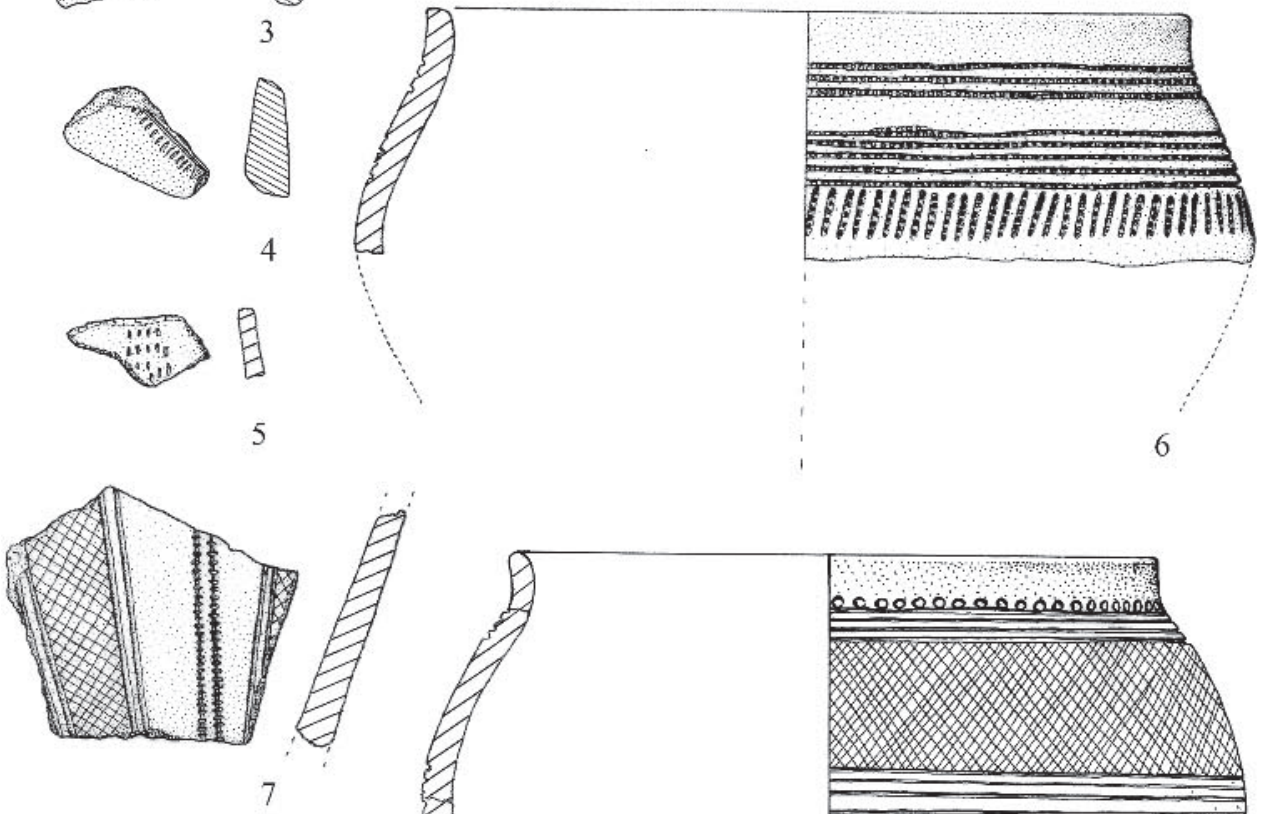

7

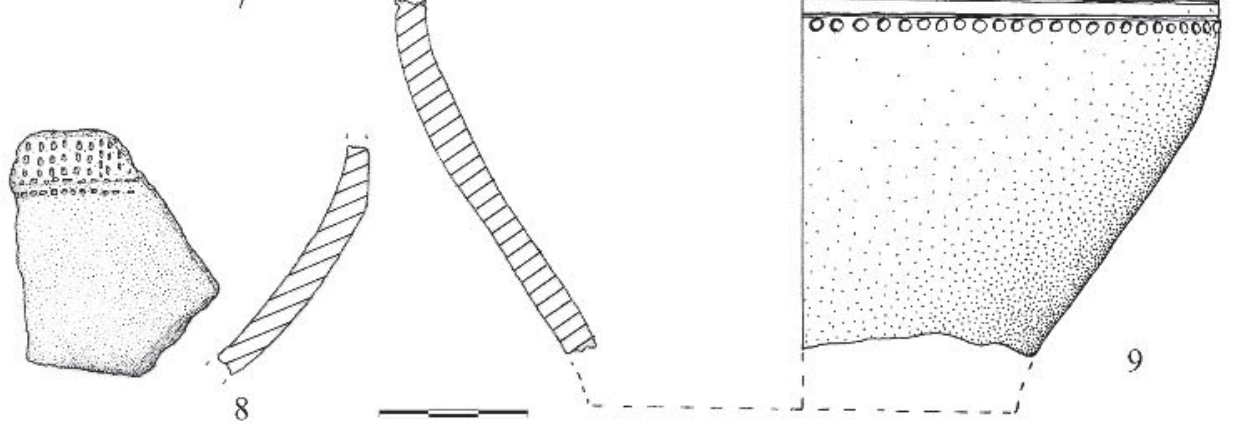

Fig. 2 - Roc d'en Gabit, Carcassonne (Aude). Formes céramiques restituables et tessons décorés dans le style épicampaniforme provenant du fossé d'enceinte de l'Âge du bronze ancien (dessin J. Vaquer, DAO M. Remicourt). Fig. 2 - Roc d'en Gabit, Carcassonne (Aude). Identifiable ceramic shapes and potsherds decorated in the "épicampaniforme» style from the Early Bronze Age ditch enclosure (drawing J. Vaquer, CAD M. Remicourt).

illustrée par un gobelet entier de la grotte de Sargel à Saint-Rome-de-Cernon en Aveyron (Thauvin-Boulestin, 1998). Il n'existe qu'un seul cas de disposition de bandes en chevrons reprenant un thème fréquent dans le Campaniforme pyrénéen dans lequel il figure en rechampi. On ne note qu'un cas de bandes orthogonales formant l'encadrement de deux panneaux décorés situés de part et d'autre d'une anse. Cette disposition ornementale est bien attestée sur des tasses et gobelets du site du Camp de Laure. Parmi les autres thèmes on note des frises de triangles hachurés qui sont eux aussi de claire dérivation campaniforme.
La structuration des motifs en bande varie très sensiblement. Parmi les bandes simples, on note des bandes simples à faisceau de lignes au peigne ou de lignes barbelées ainsi qu'une suite de motifs en X superposés

Fig. 3 (à droite) - Roc d'en Gabit, Carcassonne (Aude). Formes céramiques restituables provenant du fossé d'enceinte de l'Âge du bronze ancien (dessin J. Vaquer, DAO M. Remicourt).

Fig. 3 (in right) - Roc d'en Gabit, Carcassonne (Aude). Identifiable ceramic shapes from the Early Bronze Age ditch enclosure (drawing J. Vaquer, CAD M. Remicourt). 


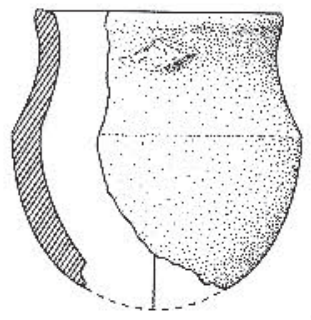

1

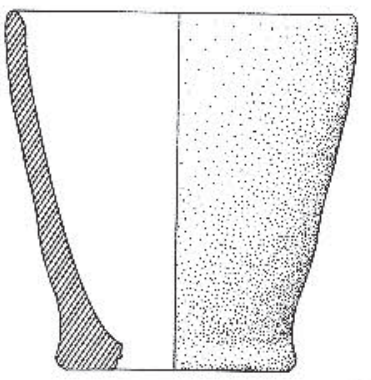

2
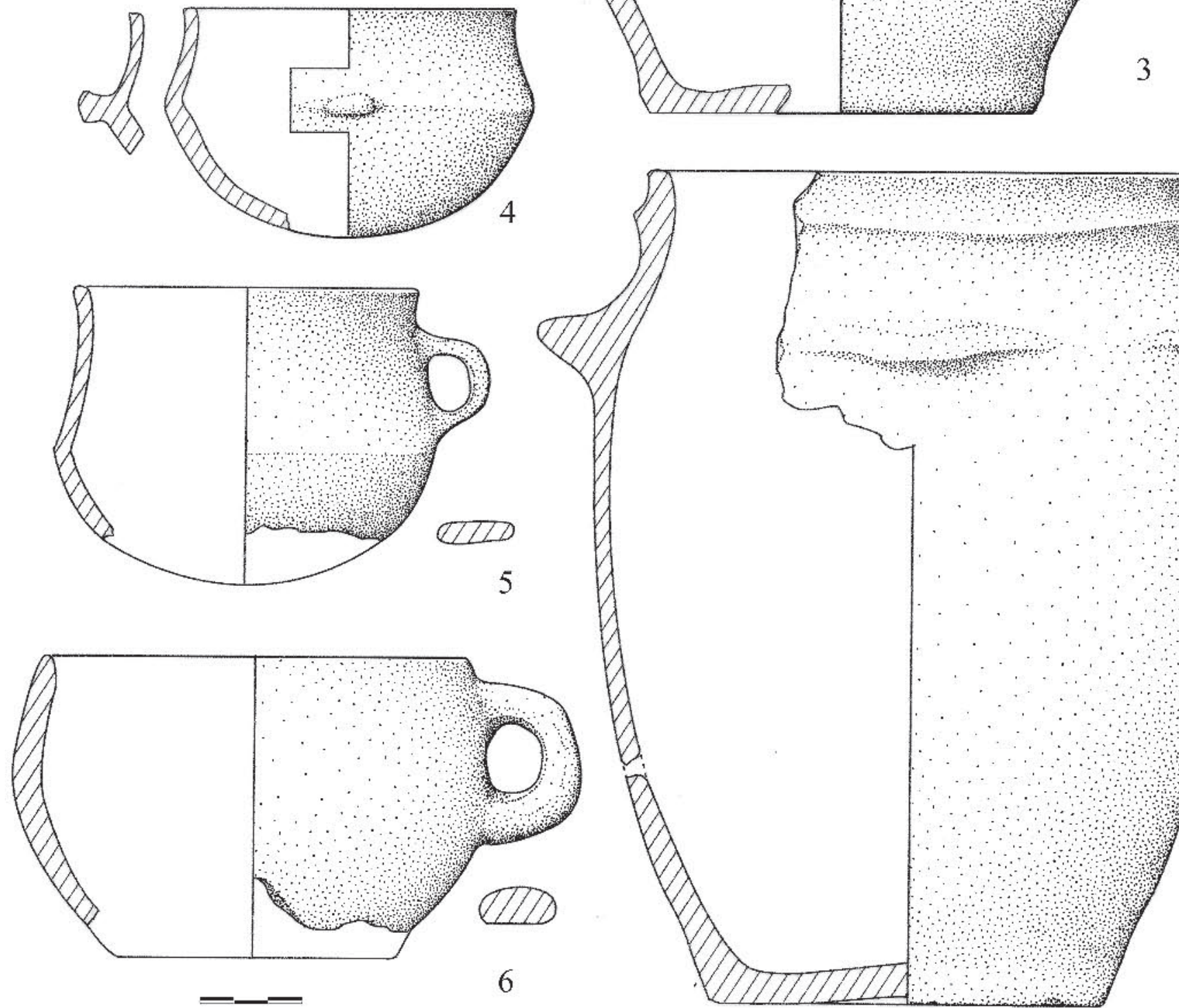

3 
ornant une anse selon une modalité connue au Camp de Laure (Courtin, 1992). Les bandes bipartites associent deux motifs superposés jointifs, comme des lignes horizontales et des hachures obliques (fig. 2, $n^{\circ} 5$ ) ou une cannelure et une frise de chevrons (fig. $2, \mathrm{n}^{\circ} 3$ ). Les bandes tripartites peuvent présenter l'association d'une bande de lignes horizontales margée par des lignes frangées (fig. 2, $n^{\circ} 2$ ). Les bandes hachurées ou croisillonnées à encadrement de lignes simples ou doubles (fig. $2, \mathrm{n}^{\circ} 6$ ) reproduisent un thème fréquent au Camp de Laure. Il y a aussi des faisceaux de lignes horizontales margées par des files d'impressions «au chalumeau », voire la superposition d'impressions au chalumeau, de lignes horizontales et de ligne frangée (fig. 2, $\mathrm{n}^{\circ} 2$ ). D'autres bandes ont une structuration quintépartite avec une bande centrale à remplissage croisillonné, ou scalariforme ou en chevrons, des bandes latérales de faisceaux de lignes et des bordures de files d'impressions au chalumeau (fig. 2, $n^{\text {os }} 1$ et 8 ). Une anse porte un motif avec une bande centrale de métopes, deux faisceaux de lignes d'encadrement et une bordure de files de traits incisés selon une modalité attestée sur le site de Derrière le Château à Géovreissiat dans l'Ain (Vital et al., 1999b).

\section{La céramique lisse et fine}

La céramique fine et soigneusement lissée est essentiellement représentée par des tasses et des petits gobelets dont les formes sont inspirées de modèles orientaux (Begleitkeramik) qui figurent dans les productions dès le Campaniforme (Besse, 1996; Strahm, 1998).

Les tasses larges et les tasses hautes forment le groupe le plus abondant et présentent une variabilité morphologique. On peut distinguer des tasses à paroi convexe (tasse en tonnelet) et fond probablement plat qui portent une anse unique sur le renflement de la panse (fig. 3, $\mathrm{n}^{\circ} 6$ ). Ce type est caractéristique du Bronze ancien, il est documenté notamment dans les séries des sites du Serre 1 à Roynac (Vital et al., 1999a et b) et dans les séries de Dallet Machal en Auvergne (Loison, 2003). D'autres variétés à paroi galbée ou carénée avec fond rond ou plat portent une anse en position haute raccordant le bord et la segmentation du profil; ce type est fréquent, il est attesté au Serre 1 de Roynac (Vital et al., 1999a et b) ou à l'aven du Gendarme à La Roque-SainteMarguerite en Aveyron (Thauvin-Boulestin, 1998). Sur d'autres exemplaires, l'anse est au-dessus de la carène sans atteindre le bord (fig. $3, n^{\circ} 5$ ), selon une disposition attestée sur de nombreux sites, comme par exemple à la grotte de Gardouch dans l'Aude (Guilaine, 1972; Gascó, 2004). Il existe aussi une tasse portant une languette sur la carène (fig. $3, n^{\circ} 4$ ), qui appartient à un type attesté à la grotte de Montou, Corbères-les-Cabanes, dans les Pyrénées-Orientales (Claustre, 1996). Cette modalité est très fréquente au Bronze ancien en Catalogne sur des tasses carénées qui sont généralement peu profondes (Equip Minferri, 1997).

Les gobelets ou godets présentent eux aussi une diversité de formes. On note la présence du gobelet tronconique à fond plat débordant (fig. $3, \mathrm{n}^{\circ} 2$ ), identique à des exemplaires trouvés dans plusieurs gisements audois, comme celui de Laval de la Bretonne à Monze (Gascó, 2004) ou de la grotte du Rec de Gruissan (Guilaine, 1996). Le gobelet caréné profond (fig. 3, n 1) est attesté; il appartient à un type du Bronze ancien présent par exemple à la grotte du Travès à Montclus dans le Gard (Roudil, 1972). On note aussi la présence d'un petit godet en tonnelet à fond plat débordant d'un type attesté au Trou du Viviès à Narbonne dans l'Aude (Guilaine, 1972).

La série comporte aussi des formes, moins bien restituables, comme des bords de coupes ou de bols à bord convexe, des petits vases tulipiformes ou subtronconiques selon la forme de leur fond. On note aussi la présence d'un couvercle ou lampe à collerette perforée d'un type fréquent au Bronze ancien dans le Midi de la France.

\section{La céramique épaisse de stockage et culinaire}

Cette céramique constitue la catégorie la mieux représentée, mais elle est très fragmentée et plus difficile à restituer, notamment pour les très grands vases.

On peut distinguer tout d'abord des vases ouverts tulipiformes munis de mamelons dont on ne possède pas le fond et qui peuvent aussi bien évoquer des formes du groupe de Véraza que du Bronze ancien. Les vases larges ou peu profonds bitronconiques ou à bord sinueux sont attestés mais très fragmentés (fig. $4, \mathrm{n}^{\circ} 2$ ). Les formes les plus fréquentes sont profondes et fermées avec des fonds plats ou discoïdes. Il s'agit essentiellement de vases bitronconiques ou en tonnelets hauts qui peuvent être de forme régulière (fig. $4, \mathrm{n}^{\circ} 1$ ) ou bien présenter un bord redressé formant un petit col plus ou moins différencié (fig. $4, \mathrm{n}^{\circ} 7$ ). Ce sont surtout les dispositifs de préhension qui permettent d'établir des distinctions au sein de ces productions courantes. On note des pots ou jarres à languettes sous le bord (fig. 4, n 6) ou bien à mamelons à languettes à la fois sur la lèvre et le haut de la panse (fig. $4, \mathrm{n}^{\circ} 1$ ) qui correspondent à des types fréquents en vallée du Rhône sur les sites de Roynac le Serre 1 ou de Chabrillan Saint-Martin (Vital, 2004). Un autre mode de préhension courant est constitué par un ou deux cordons lisses horizontaux placés sur le bord ou en dessous et associés ou non à des languettes (fig. $3, \mathrm{n}^{\circ} 7$ et fig. 4, n 5). Ces formes simples sont banales dans le Bronze ancien du Midi et rattachées à la culture du Rhône, si l'on retient comme typiques les formes semblables des dépôts du site du Petit-Chasseur à Sion en Suisse (Bocksberger, 1976; Gallay, 1986) ou de Dallet Machal en Puy-deDôme (Loison, 2003). Elles sont très bien représentées

Fig. 4 (à droite) - Roc d'en Gabit, Carcassonne (Aude). Formes céramiques restituables provenant du fossé d'enceinte de l'Âge du Bronze ancien (dessin J. Vaquer, DAO M. Remicourt).

Fig. 4 (in right) - Roc d'en Gabit, Carcassonne (Aude). Identifiable ceramic shapes from the Early Bronze Age ditch enclosure (drawing J. Vaquer, CAD M. Remicourt). 


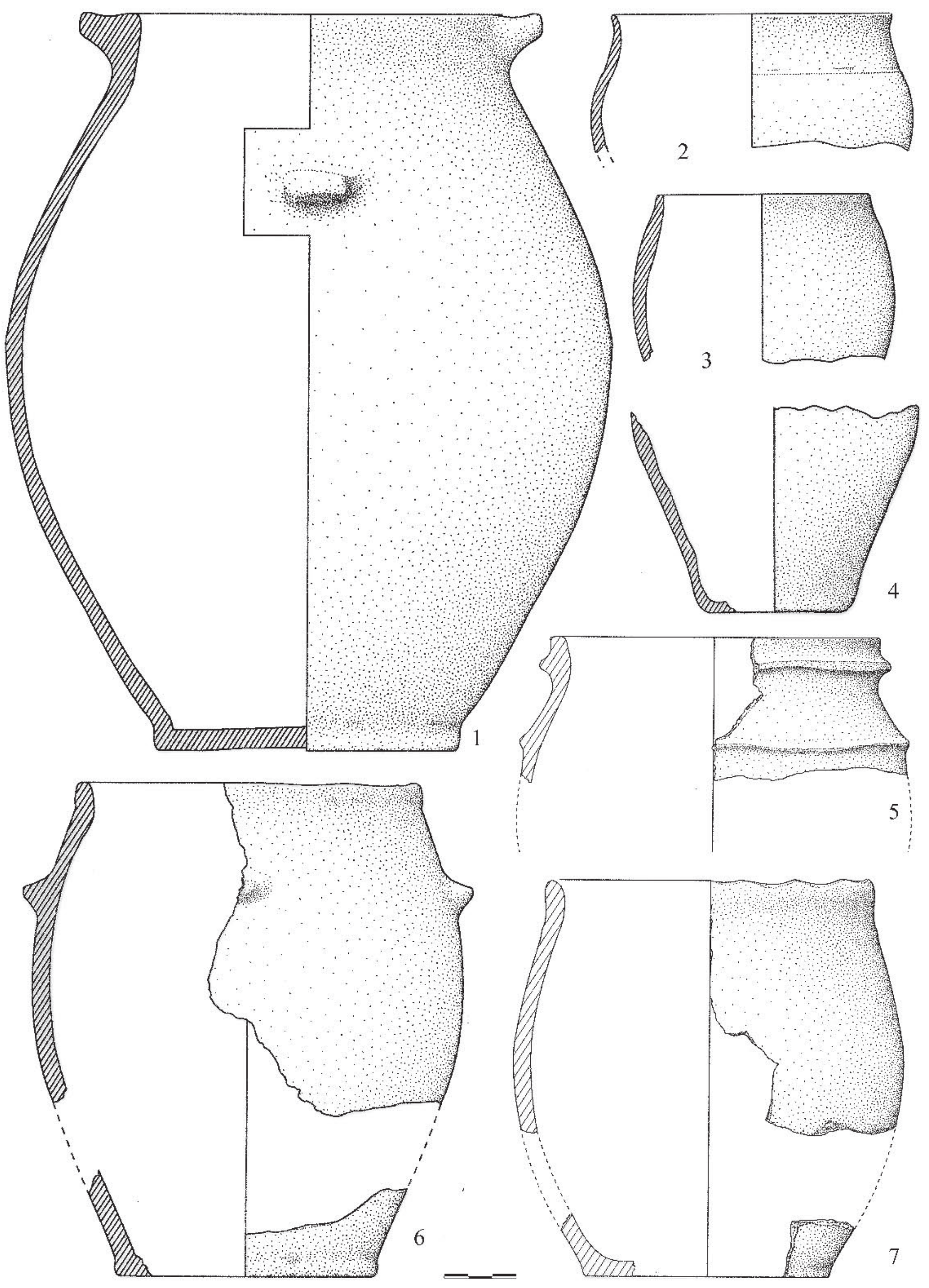


aussi dans le style du Camp de Laure si on tient compte des nombreux exemplaires du site éponyme (Courtin, 1975). D'autres systèmes de préhension sont constitués par des anses en ruban placées au-dessus du renflement de la panse (fig. $3, n^{\circ} 3$ ), selon une modalité bien connue à Roynac le Serre 1 dans la Drôme ou à la grotte du Ranc d'Aven à Grospierre en Ardèche (Vital, 2004). On peut citer le cas d'un vase en tonnelet qui porte une anse en ruban se poursuivant par un cordon lisse horizontal ceinturant le haut de la panse, selon une modalité attestée à Roynac le Serre 1 dans la Drôme (Vital, 2004).

\section{LA CÉRAMIQUE DE ROC D'EN GABIT DANS SON CONTEXTE RÉGIONAL}

Il est généralement admis que la céramique du début du Bronze ancien présente dans le Midi de la France un syncrétisme de récurrences chalcolithiques et de legs campaniformes régionaux (Gascó, 2004), auxquels s'ajoutent de nouveaux caractères orientaux originaires d'Europe centrale et d'Italie (Vital, 1996 et 2004). Dans la zone pyrénéenne, on peut envisager d'autres apports venant de la péninsule Ibérique (Claustre, 1996) ou propres à la façade atlantique de l'Europe (Roussot-Larroque, 1996).

La composante héritée du Chalcolithique local, c'est-à-dire du groupe de Véraza, existe de façon ténue dans la série céramique de Roc d'en Gabit, mais elle n'est pas clairement interprétable comme un legs ou une récurrence. Nous avons constaté effectivement la présence de vases à fond rond, principalement des bols, des coupes et des gobelets ou vases tulipiformes munis de mamelons ou de languettes sous le bord. Il pourrait s'agir de caractères relevant des traditions du Vérazien, mais il est impossible de savoir s'il s'agit d'éléments véritablement contemporains de ceux du Bronze ancien dans la mesure où des intrusions de mobiliers plus anciens et des recoupements de structures sont prouvés sur le site. La persistance de traditions potières issues du Vérazien jusqu'au début du Bronze ancien apparaît donc douteuse à Roc d'en Gabit. Aucun vase présentant des caractères mixtes n'a pu être individualisé sur le gisement, contrairement à ce qui a pu être observé dans certaines séries catalanes (séries de Minferri, Can Roqueta et Poliesportiu de la UAB) (Equip Minferri, 1997). Si l'on retient l'hypothèse de la persistance de la culture vérazienne jusqu'au Bronze ancien, cela implique de la considérer comme contemporaine du Campaniforme pyrénéen. Or ce synchronisme n'a jamais pu être prouvé de façon claire. On constate plutôt que l'horizon campaniforme maritime peut apparaître, déjà de façon autonome, en succédant au Vérazien sur le site de Mourral à Trèbes (Vaquer, 1998). Le Campaniforme pyrénéen est quant à lui très fortement représenté dans la région sous une forme indépendante, comme l'illustre le site de Médor à Ornaisons dans l'Aude (Guilaine et al., 1989). Il ne présente sur ce site aucun syncrétisme net avec le Vérazien auquel il semble succéder avec une nette rupture des traditions potières.
La composante épicampaniforme est très prégnante dans la série de Roc d'en Gabit. Elle s'exprime aussi bien dans la céramique décorée relevant du style barbelé ou style du Camp de Laure que dans la céramique non décorée, dont de nombreux éléments dérivent de la céramique commune des groupes campaniformes maritimes et régionaux. Nous avons réalisé des tableaux comparatifs d'occurrences de types, afin de tenter de cerner plus précisément ce qui peut dériver des groupes campaniformes antérieurs et de discriminer les nouveaux caractères trahissant des liens avec d'autres horizons épicampaniformes. Pour le Campaniforme rhodano-provençal, nous avons choisi deux sites gardois : le Bois Sacré à Saint-Côme-et-Maruéjols (Roudil et al., 1974) et la station de Maupas à Calvisson (Roger et al., 1988; Lemercier, 2004). Pour le Campaniforme pyrénéen, nous avons choisi deux sites audois : Embusco 3 à Mailhac (Guilaine, 1967; Besse, 1996) et le fossé de Médor à Ornaisons (Guilaine et al., 1989). Pour l'étape ancienne du Bronze ancien, nous avons retenu les séries les plus représentatives du domaine provençal, c'est-à-dire celle du Camp de Laure au Rove (Courtin, 1975; Lemercier, 2004) et celle du Clos Marie-Louise à Aix-en-Provence dans les Bouches-du-Rhône (Vignaud, 2002; Lemercier, 2004). Pour le Languedoc, nous avons retenu la série de Parignoles à La Livinière dans l'Hérault (Ambert, 2003) et pour l'Auvergne la série du Tourteix à Orcet dans le Puy-de-Dôme (Loison, 2003).

Le tableau comparatif d'occurrences des formes révèle que la plupart des éléments recensés à Roc d'en Gabit se retrouvent dans les autres séries de comparaison, avec toutefois des variations dans le nombre d'occurrences communes (fig. 5). Ces variations peuvent s'expliquer soit par une véritable différence de composition typologique, soit par une inégalité de la taille des effectifs disponibles. Il faut noter en premier lieu que la majeure partie du fonds typologique présent à Roc d'en Gabit existe sur les quatre sites de référence du Bronze ancien méridional. Ce ne sont pas moins de 22 éléments présents à Roc d'en Gabit qui sont attestés sur les autres sites réunis, soit $22 / 25=88 \%$. De plus, les trois éléments manquants sont assez rares, mais connus sur d'autres sites bien datés : les bouchons à collerette sont attestés notamment à la grotte du Travès à Montclus dans le Gard (Roudil, 1972), les écuelles carénées larges à anse unique ou à mamelon sur la carène ont été signalées dans quelques sites languedociens et pyrénéens du Bronze ancien, comme la grotte de Montou à Corbères-les-Cabanes (Claustre, 1996) ou la grotte du Chien à Cesseras dans l'Hérault (Guilaine, 1972). Il ressort de ces comparaisons que la série de Roc d'en Gabit a une composition typologique tout à fait classique par rapport à l'horizon du début du Bronze ancien du Midi français.

L'autre constatation notable qui découle de ces comparaisons est que plus de la moitié des critères recensés pour ces assemblages du début du Bronze ancien existe aussi dans les assemblages de céramiques décorées et communes des styles régionaux du Campaniforme du Midi de la France (15 critères sur 25, soit $60 \%$ ). Ce constat confirme bien la dérivation du style 


\begin{tabular}{|c|c|c|c|c|c|c|c|c|c|c|c|c|c|}
\hline & 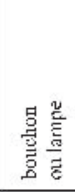 & 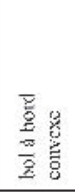 & 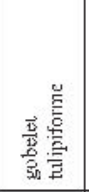 & 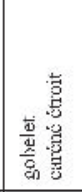 & 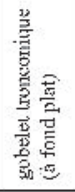 & 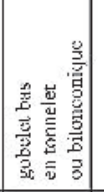 & 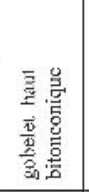 & 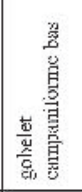 & 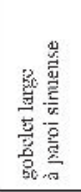 & 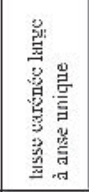 & 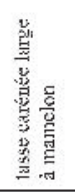 & 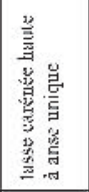 & 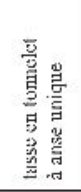 \\
\hline $\begin{array}{l}\text { Station de Roc d'en Gabit } \\
\text { Carcassonne } \\
\text { Aulde }\end{array}$ & $\bigodot_{\approx}$ & $\varpi$ & 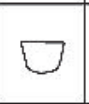 & $\theta$ & $\square$ & $\square$ & $\square$ & $\square$ & $\square$ & $\theta$ & $\Theta$ & $\theta$ & $\square^{\circ}$ \\
\hline $\begin{array}{l}\text { Station de Bois Sacré } \\
\text { Saint-Cóme-et-Marújols } \\
\text { Gard }\end{array}$ & & 0 & 0 & & 0 & 0 & 0 & 0 & 0 & 0 & & & 0 \\
\hline $\begin{array}{l}\begin{array}{l}\text { Station de Maupas } \\
\text { Calvisson } \\
\text { Gard }\end{array} \\
\end{array}$ & & 0 & & & & & & 0 & 0 & & & & 0 \\
\hline $\begin{array}{l}\text { Station d't'inbusco } 3 \\
\text { Mailhac } \\
\text { Aude }\end{array}$ & & 0 & 0 & & & & & 0 & & & & & \\
\hline $\begin{array}{l}\text { Station de Mèdor } \\
\text { Ornaisons } \\
\text { Aulc } \\
\end{array}$ & 0 & 0 & & & & 0 & 0 & 0 & 0 & & & & \\
\hline $\begin{array}{l}\text { Stutim de Parignoles } \\
\text { La Livinière } \\
\text { Hérault }\end{array}$ & & 0 & & & & 0 & & 0 & 0 & & & 0 & 0 \\
\hline $\begin{array}{l}\text { Camp de Laure } \\
\text { Rove } \\
\text { Bouches-du-Rhône }\end{array}$ & & & 0 & 0 & 0 & 0 & 0 & 0 & ○ & & & ○ & 0 \\
\hline $\begin{array}{l}\text { Clos Marie-Louise } \\
\text { Aix-en-Provencence } \\
\text { Bouchles-du-Rhône }\end{array}$ & & 0 & & & & & & & & & & 0 & 0 \\
\hline $\begin{array}{l}\text { Le Tourteix } \\
\text { Orcet } \\
\text { Puy-de-Dôme }\end{array}$ & & & & 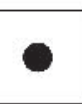 & & 0 & & & & & & 0 & \\
\hline
\end{tabular}

\begin{tabular}{|c|c|c|c|c|c|c|c|c|c|c|c|c|}
\hline & 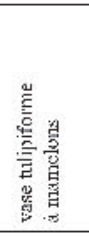 & 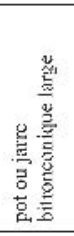 & 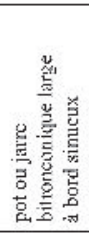 & 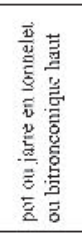 & 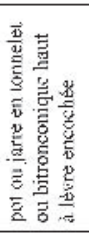 & 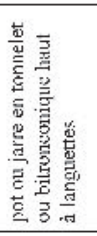 & 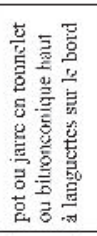 & 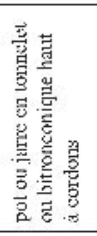 & 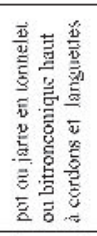 & 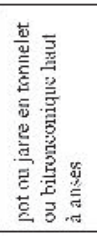 & 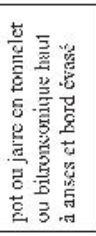 & 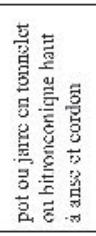 \\
\hline $\begin{array}{l}\text { Station de Roc d'en Gabit } \\
\text { Carcassome } \\
\text { Audc }\end{array}$ & $0\}$ & & & & & 0 & & & & & & \\
\hline $\begin{array}{l}\text { Station de Bois Sacré } \\
\text { Saint-Cômo-ct-Maruéjols } \\
\text { Garrl }\end{array}$ & & & 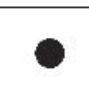 & & 0 & & 0 & 0 & & & & \\
\hline $\begin{array}{l}\text { Station de Maupas } \\
\text { Calvissem } \\
\text { Garde }\end{array}$ & & 0 & ○ & & & & & 0 & & & & \\
\hline $\begin{array}{l}\text { Station d'Emblusco } 3 \\
\text { Maililac } \\
\text { Auld }\end{array}$ & & - & 0 & & 0 & & & 0 & & & & \\
\hline $\begin{array}{l}\text { Sation de Médor } \\
\text { Ornaisists: } \\
\text { Aude. }\end{array}$ & & & ○ & & ○ & & & 0 & & & & \\
\hline $\begin{array}{l}\text { Station de Parignoles } \\
\text { La Livinierese } \\
\text { IIérault }\end{array}$ & & & & & ○ & & & 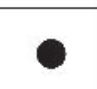 & & & 0 & \\
\hline $\begin{array}{l}\text { Camp de Laure } \\
\text { Rove } \\
\text { Bouches-du-Rhône }\end{array}$ & & 0 & & 0 & 0 & 0 & 0 & 0 & 0 & 0 & 0 & \\
\hline $\begin{array}{l}\text { Clos Marie-Louise } \\
\text { Aix-en-Provence } \\
\text { Bouches-du-Rhône }\end{array}$ & & 0 & & & 0 & 0 & & 0 & & 0 & & \\
\hline $\begin{array}{l}\text { Le Tourteix } \\
\text { Orcet } \\
\text { Puy-de-Dóme }\end{array}$ & 0 & & 0 & 0 & & 0 & & 0 & 0 & 0 & 0 & \\
\hline
\end{tabular}

Fig. 5 - Tableau d'occurrences des formes céramiques de Roc d'en Gabit et d'autres ensembles de l'Âge du bronze ancien dans le Sud de la France (conception et DAO M. Remicourt et J. Vaquer).

Fig. 5 - Occurrence table for ceramic forms from Roc d'en Gabit and other assemblages of the Early Bronze Age in the south of France (design and CAD M. Remicourt and J. Vaquer). 


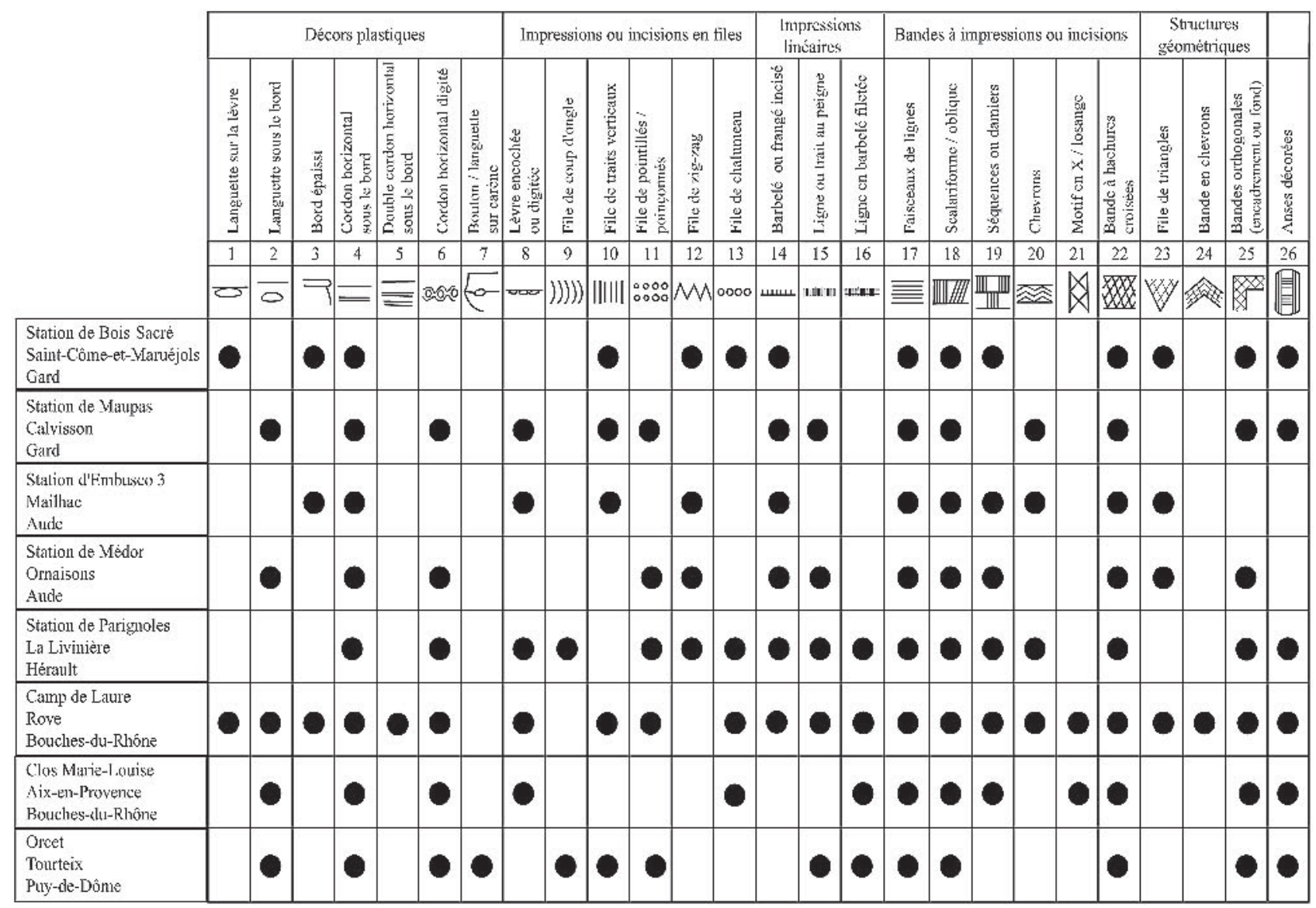

Fig. 6 - Tableau d'occurrences des décors céramiques de Roc d'en Gabit et d'autres ensembles de l'Âge du bronze ancien dans le Sud de la France (conception et DAO M. Remicourt et J. Vaquer).

Fig. 6 - Occurrence table for ceramic decorations from Roc d'en Gabit and other Early Bronze Age assemblages in the south of France (design and CAD M. Remicourt and J. Vaquer).

Bronze ancien à partir de ces deux groupes régionaux campaniformes, comme cela a été envisagé par de nombreux auteurs. Il faut noter à ce sujet que les styles pyrénéen et rhodano-provençal se distinguent surtout par les décors et beaucoup moins par les formes des récipients. En ce qui concerne les formes, le changement concerne la disparition de quelques types censés être d'inspiration ibérique comme les cazuelas ou les bols et les coupelles à fond ombiliqué (une particularité de cette époque qui transfère à la céramique une technique métallurgique). Il se traduit aussi par la disparition de certaines particularités comme les perforations traversières sous le bord des gros vases. Dans ce dernier cas, il s'agit plutôt d'un caractère d'origine nordorientale ou italique, si l'on se fie à sa présence dès le style «maritime» à Champ-Vully à Rances (Vaud, Suisse), à Noir Bois, Alle (Jura) ou à Bulimaco-Cilea, Cesto-Fiorentino, en Toscane (Besse, 1996; Sarti et Martini, 1993). Le renouvellement par rapport aux groupes campaniformes régionaux concerne essentiellement les gobelets ou tasses carénées hautes à anse et parfois à mamelons contre les anses (imitant peut-être des rivets de modèles métalliques). Ce sont des types inspirés de modèles des zones orientales (domaine nord-alpin et Italie) où ils apparaissent dès le Campaniforme (Begleitkeramik). Le renouvellement concerne aussi les gros vases de stockage bitronconiques ou en tonnelet pourvus de cordons lisses, de languettes et d'anses en ruban, qui peuvent eux aussi copier des types orientaux plus précoces ou bien résulter d'une évolution de la céramique commune campaniforme de l'aire italo-franco-suisse.

Le tableau comparatif des décors est encore plus explicite que celui des formes puisqu'une grande partie des 26 caractères attestés à Roc d'en Gabit se retrouvent dans les quatre séries (fig. 6) que nous avons sélectionnées pour le Bronze ancien méridional (Parignoles, Camp de Laure, Clos Marie-Louise, Orcet). Il faut noter surtout une concordance très forte avec la série du Camp de Laure qui est la plus représentative en raison de sa richesse. On constate que 23 des 26 caractères de la série de Roc d'en Gabit sont attestés au Camp de Laure, ce qui justifie le rattachement de Roc d'en Gabit au style défini sur ce site. La seconde constatation que l'on peut tirer de ce tableau est que 20 des 26 caractères attestés à Roc d'en Gabit existent déjà dans les séries campaniformes régionales (Bois Sacré, Maupas, Embusco 3, Médor). Cela nous parait justifier pleinement l'expression «épicampaniforme» utilisée pour le style du Camp de Laure, d'autant plus que certains caractères absents de nos quatre séries de comparaison existent sur d'autres 
sites. Le critère 5 existe à la grotte Murée de Montpezat dans les Alpes-de-Haute-Provence, il existe aussi avec le critère 9 à Géovreissiat dans l'Ain, le critère 21 est attesté à la station de Toupiguières à Grans dans les Bouches-du-Rhône et le critère 24 existe à la Brégoule à Soyons en Ardèche (Besse, 2003; Lemercier, 2004). Il y a donc peu de critères qui participent au renouvellement du Bronze ancien, si ce n'est la généralisation de la technique d'impression à la tige souple filetée et des changements de fréquence des motifs ou de leur agencement sur les vases.

Pour affiner ces comparaisons, nous avons tenu compte aussi des éléments différents de ceux attestés à Roc d'en Gabit et calculé le coefficient de Jaccard entre la série de Roc d'en Gabit et nos séries de comparaison. L'indice de similarité ou coefficient de communauté de Jaccard SJij (1908) est appliqué aux données binaires (présence-absence) et est couramment utilisé en écologie (phytosociologie, entomologie) pour tester l'homogénéité endogène d'un syntaxon élémentaire (qui se traduit par l'existence d'espèces constantes). Il permet de mesurer la proportion d'espèces communes à deux relevés $i$ et $j$ par rapport au total des espèces qu'ils contiennent ensemble (Gillet, 2000). Cet indice a ensuite été étendu à d'autres disciplines comme la linguistique ou l'archéologie :

\section{$\mathrm{SJ} i j=\mathrm{a} / \mathrm{a}+\mathrm{b}+\mathrm{c}$}

$\mathrm{a}=$ nombre d'espèces communes au relevé $i$ et au relevé $j$

$\mathrm{b}=$ nombre d'espèces présentes seulement dans le relevé $i$

$\mathrm{c}=$ nombre d'espèces présentes seulement dans le relevé $j$

Théoriquement, un couple de relevés est considéré comme appartenant à un ensemble très homogène s'il présente un coefficient de Jaccard proche de 1. On considère qu'il appartient à un ensemble homogène si le coefficient de Jaccard est supérieur ou égal à 0,5 . Dans la pratique, et pour les couples quantitativement inégaux, il est admis par les utilisateurs que si cet indice est supérieur à 0,3 les séries peuvent faire partie d'un même ensemble.

Les données chiffrées qui sont obtenues montrent à nouveau une forte ressemblance entre la série de Roc d'en Gabit et celle du Camp de Laure, qui se traduit aussi bien au niveau des formes des vases que des décors (fig. 7). Avec des coefficients de Jaccard situés entre 0,6 et 0,7 , on peut conclure que les deux séries appartiennent à un ensemble bien homogène. Pour les sites de Parignoles et de Clos Marie-Louise, les valeurs des coefficients sont plus élevées pour les décors que pour les formes. Le caractère limité de ces deux séries est sans doute en cause ici et l'on peut retenir que ces séries appartiennent elles aussi au même ensemble

\begin{tabular}{|c|c|c|c|c|c|c|c|c|c|c|c|c|}
\hline & 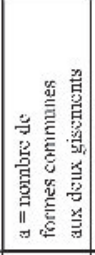 & 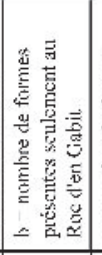 & 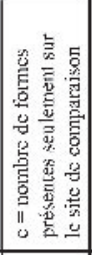 & 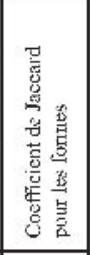 & 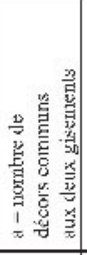 & 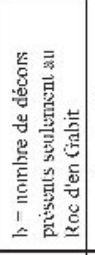 & 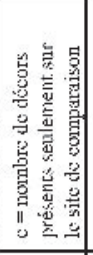 & 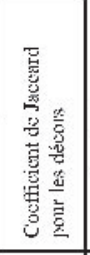 & 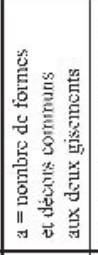 & 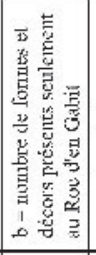 & 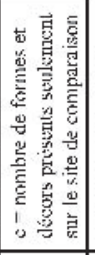 & 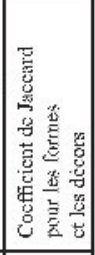 \\
\hline $\begin{array}{l}\text { Station de Bois Sacré } \\
\text { Saint-Côme-et-Maruẹjols } \\
\text { Gard }\end{array}$ & 13 & 12 & 8 & 0,39 & 14 & 12 & 6 & 0,44 & 27 & 24 & 14 & 0,42 \\
\hline $\begin{array}{l}\text { Station de Maupas } \\
\text { Calvisson } \\
\text { Gard }\end{array}$ & 7 & 18 & 6 & 0,23 & 14 & 12 & 8 & 0,41 & 21 & 30 & 14 & 0,32 \\
\hline $\begin{array}{l}\text { Stution d'Eunlyusce } 3 \\
\text { Maillhic } \\
\text { Aucklc }\end{array}$ & 7 & 18 & 4 & 0,24 & 13 & 13 & 5 & 0,42 & 20 & 31 & 9 & 0,33 \\
\hline $\begin{array}{l}\text { Station de Médor } \\
\text { Ornaisons } \\
\text { Aude }\end{array}$ & 9 & 16 & 10 & 0,26 & 14 & 12 & 11 & 0,38 & 23 & 28 & 21 & 0,32 \\
\hline $\begin{array}{l}\text { Station de Parignoles } \\
\text { Lia Livinière } \\
\text { Hératult. }\end{array}$ & 9 & 16 & 2 & 0,33 & 17 & 9 & 5 & 0,55 & 26 & 25 & 7 & 0,45 \\
\hline $\begin{array}{l}\text { Gamp de Liute } \\
\text { Rove } \\
\text { Boushes-du-Rhône }\end{array}$ & 19 & 6 & 5 & 0,63 & 23 & 3 & 7 & 0,70 & 42 & 9 & 12 & 0,67 \\
\hline $\begin{array}{l}\text { Clos Marie-Louise } \\
\text { Aix-en-Prowence } \\
\text { Bouches-du-Rhône } \\
\end{array}$ & 8 & 17 & 4 & 0,28 & 13 & 13 & 0 & 0,50 & 21 & 30 & 4 & 0,38 \\
\hline $\begin{array}{l}\text { Le'Tourteix } \\
\text { Orecl } \\
\text { Puy-de-Dôme }\end{array}$ & 13 & 12 & 8 & 0,39 & 14 & 12 & 7 & 0,42 & 27 & 24 & 15 & 0,41 \\
\hline
\end{tabular}

Fig. 7 - Tableau de décompte des occurrences de formes et de décors de Roc d'en Gabit et d'autres ensembles de l'Âge du bronze ancien dans le Sud de la France et coefficient de Jaccard (conception et DAO M. Remicourt et J. Vaquer).

Fig. 7 - Breakdown of occurrences of shapes and decorations from Roc d'en Gabit and other Early Bronze Age assemblages in the south of France and the Jaccard coefficient (design and CAD M. Remicourt and J. Vaquer). 
homogène. Ce problème d'effectif ne semble pas se poser dans le cas de la série de Tourteix qui paraît plus diversifiée et sans doute constituée sur une durée plus longue. Les valeurs du coefficient de Jaccard obtenues pour les sites campaniformes sont toutes supérieures à 0,3 sur l'ensemble des critères, ce qui indique l'existence d'un lien phylétique probable avec les séries du début du Bronze ancien. Il ne semble pas exister de différence importante dans les contributions entre les séries du faciès rhodano-provençal et celles du faciès pyrénéen. S'il est admis que le style barbelé est marqué par de nouveaux apports orientaux et italiques, seule la valeur un peu plus élevée obtenue pour la série du Bois Sacré pourrait témoigner de l'adoption plus précoce des caractères du Bronze ancien en zone basrhodanienne.

Étant donné la localisation de Roc d'en Gabit par rapport au domaine pyrénéen, il est logique de regarder si la série de critères caractérisant la céramique de ce site peut avoir des correspondances avec le Bronze ancien d'Outre-Pyrénées, c'est-à-dire principalement avec le Bronze ancien du Nord-Est ibérique, voire avec le Bronze ancien valencien.

Si l'on examine le corpus des formes, on constate évidemment des parentés qui concernent la généralisation des vases à fond plat ou l'adoption de formes carénées dans la catégorie des vases de service ou l'adoption des formes en tonnelet ou biconiques dans la céramique culinaire ou de stockage. On constate aussi l'existence de legs campaniformes dans les décorations de la céramique fine (styles d'Arboli et de Boquique) et un développement des décors plastiques impressionnés. Il faut évidemment dépasser ce niveau de convergences générales pour chercher des liens plus concrets entre les groupes culturels.

En ce qui concerne les formes, nous avons déjà signalé que les bouchons à collerette existent déjà dans le Campaniforme pyrénéen à Médor. Ils sont attestés sous des formes très semblables des deux côtés des Pyrénées au début du Bronze ancien, comme le prouvent les exemplaires typiques des grottes de Vallmayor (Banyeres) et d'Arboli $\mathrm{CH}$, près de Reus en Catalogne (Vilaseca, 1973). La perduration de coupes, de bols à fond plat et bord convexe ou de vases tulipiformes dans les séries du Bronze ancien est probablement aussi un autre legs campaniforme qui est mieux marqué en zone pyrénéenne que provençale. Ces formes ouvertes sont en effet très présentes dans les grands sites du Bronze ancien catalan, comme Can Roqueta II à Sabadell (Gonzalez Marcen et al., 1999) ou comme Minferri (Equip Minferri, 1997), voire les silos du Poliesportiu de la UAB à Barcelone (Francès y Farré, 1993). Un autre caractère rare en zone rhodano-provençale et fréquent en zone pyrénéenne est constitué par les écuelles carénées larges dépourvues d'anses en ruban mais portant des mamelons sur la carène. Ces formes sont sans doute dérivées des cazuelas campaniformes, elles sont présentes sur la plupart des sites sud-pyrénéens et se retrouvent aussi à Montou (Claustre, 1996). Pour les grands vases, d'autres formes plutôt rares dans le Midi français sont fréquentes en Catalogne; c'est le cas en particulier pour les pots bitronconiques ou globuleux larges à bord convexe ou sinueux qui sont typiques du style d'Arboli et d'autres groupes pyrénéens. C'est le cas aussi des jarres hautes à bord sinueux avec mamelons ou impressions sur la lèvre que l'on trouve dans la plupart des sites du Bronze ancien catalan.

En ce qui concerne les décors, il existe évidemment des parentés entre les séries du Midi français et celles de Catalogne pour tous les décors épicampaniformes. On rappellera toutefois que la perduration de décors au peigne est attestée en domaine catalan sur des ensembles bien datés du Bronze ancien comme les silos du Poliesportiu de la UAB. Il faut noter surtout à Roc d'en Gabit quelques éléments d'influence catalane probable. Un décor de chevron frangé de traits orthogonaux (barbelé ou frangé incisé) reproduit un thème typique du style d'Aboli (fig. $2, \mathrm{n}^{\circ} 4$ ). Un autre décor de cannelure surmonté d'une file de zigzags reproduit un thème très fréquent dans le Bronze ancien valencien (fig. $2, n^{\circ} 3$ ). Ces influences transpyrénéennes paraissent toutefois très limitées dans la série du Roc d'en Gabit.

\section{CONCLUSION}

Le site de Roc d'en Gabit a livré une série céramique représentative du début du Bronze ancien qui provient de structures d'habitat à comblement rapide (le fond d'un fossé d'enceinte et quelques fosses). Cette série comporte trois composantes : une vaisselle fine décorée dans le style barbelé, une vaisselle fine lisse dominée par les gobelets et tasses ou pichets carénés à une seule anse et une céramique grossière destinée au stockage et à la cuisson. Les héritages des cultures chalcolithiques locales paraissent très douteux dans la série de Roc d'en Gabit dans la mesure où des intrusions d'éléments plus anciens sont prouvées. Les comparaisons qui ont été effectuées, tant au niveau des formes que des appendices de préhension ou des décors, permettent d'attribuer la série de Roc d'en Gabit au style du Camp de Laure tel qu'il a été défini par J. Courtin et J. Bill. Les aspects pouvant évoquer des influences ibériques sont très ténus dans la série. Il faut retenir que les concordances typologiques sont très nombreuses avec les séries du site éponyme du Camp de Laure et sont très fortes avec d'autres séries moins représentatives de ce même groupe culturel, au point que l'on peut considérer cette tradition culturelle comme homogène sur l'ensemble du Midi méditerranéen français. D'autres comparaisons ont été effectuées avec des ensembles représentatifs des deux faciès campaniformes antérieurs du Midi de la France : elles confirment que la majeure partie des caractères du style de Camp de Laure existait auparavant aussi bien dans le groupe rhodano-provençal que dans le groupe pyrénéen. La tradition potière du groupe du Camp de Laure reprend des formes de la céramique commune des groupes régionaux en ajoutant des gobelets, tasses et pichets carénés à une anse, d'origine orientale. La décoration se concentre sur ces formes avec des motifs et thèmes qui sont recomposés et traités principalement 
avec des matrices crantées (peigne, tige souple filetée) privilégiant les lignes barbelées ou frangées, les bandes à hachures croisées et les bordures à impressions de chalumeau. Ce nouveau style est très normalisé sur une large zone couvrant l'Italie du Nord et le Midi de la France. Il marque la fin des différences de style qui existaient au moment de l'épanouissement des faciès campaniformes régionaux. L'homogénéisation pourrait être due à une suprématie des relations et influences orientales que l'on souhaiterait pouvoir mieux examiner dans les productions métalliques de cette époque. Elle correspond sans doute aussi à une période de croissance du nombre des habitats, ou tout au moins à une période de restructuration sociale qui se traduit par l'apparition de nouveaux sites fortifiés dont Roc d'en Gabit est un bon exemple pour le bassin de l'Aude.

\section{RÉFÉRENCES BIBLIOGRAPHIQUES}

AMBERT P. (2003) - Contribution à l'étude du Campaniforme du Languedoc central méridional, Bulletin de la Société préhistorique française, t. $100, \mathrm{n}^{\circ} 4$, p. 715-732.

BAILLOUD G. (1966) - La civilisation du Rhône et le Bronze ancien du Midi de la France, Revue archéologique de L'Est et du Centre-Est, t. XVII, fasc. 3-4, p. 131-164.

BARGE-MAHIEU H. (1992) - Le Campaniforme dans le Midi de la France : origine et identité culturelle, catalogue d'exposition, Orgon, juil.-sept. 1992, éd. EPA, Marseille, 96 p.

BESSE M. (1996) - Le Campaniforme en France. Analyse de la céramique d'accompagnement, BAR International Series, 635, éd. BAR, Oxford, $189 \mathrm{p}$

BESSE M. (2003) - L'Europe du $3^{e}$ millénaire avant notre ère : les céramiques communes au Campaniforme, Cahiers d'Archéologie romande, $\mathrm{n}^{\circ}$ 94, éd. BHV, Lausanne, 223 p.

BILL J. (1973) - Die Glockenbecherkultur und die frühe Bronzezeit im französischen Rhonebecken und ihre Beziehungen zur Südwestschweiz, Antiqua 1, Société suisse de Préhistoire et d'Archéologie, Bâle.

BILL J. (1976) - Die Frühphase der Glockenbecherkultur in OstFrankreich, Glockenbechersymposion, Oberried, 1974, Unieboek b.v., Bussum, p. 333-349.

BILL J. (1977) - La céramique du début du Bronze ancien dans le Midi de la France, Actes du congrès préhistorique de France, $X X^{e}$ session, Provence-Martigues, 1974, éd. SPF, Paris, p. 34-39.

BOCKSBERGER O.-J. (1976) - Le site préhistorique du Petit-Chasseur (Sion, Valais), 1. Le dolmen MVI, Cahiers d'Archéologie romande, $\mathrm{n}^{\circ} 6$, éd. BHV, Lausanne, 2 vol., 211 et $151 \mathrm{p}$.

CLAUSTRE F. (1996) - Le Bronze ancien en Roussillon, in C. Mordant et O. Gaiffe dir., Cultures et sociétés de l'Âge du bronze ancien en Europe, Actes du $117^{e}$ congrès national des Sociétés savantes, Clermont-Ferrand, 1992, éd. du CTHS, Paris, p. 387-399.

COURTIN J. (1975) - Un habitat fortifié du Bronze ancien en BasseProvence : le Camp de Laure, commune du Rove (Bouches-duRhône), Bulletin du Muséum d'histoire naturelle de Marseille, t. XXXV, p. 217-240

COURTIN J. (1992) - Le Camp de Laure, Le Rove (Bouches-du-Rhône), in $\mathrm{H}$. Barge dir., Le Campaniforme dans le Midi de la France, origine et identité culturelle, éd. EPA, Marseille, p. 45-48.

EQUIP MINFERRI (AGUSTI B., ALONSO N., BORRÀS H. et al.) (1997) - Noves dades per a la caracterització dels assentaments a l'aire lliure durabnt la primera meitat del II milleni cal. BC: primers resultats de les excavacions en el jaciment de Minferri (Juneda, Les Garrigues), Revista d'Arqueòlogia del Ponent, $\mathrm{n}^{\circ}$ 7, p. 161-211.

FRANCÈS I FARRÉ J. (1993) - Les estructures del bronze antic del poliesportiu de la UAB: primers resultats, Limes, ${ }^{\circ} 3$, p. 5-24.

GALLAY A. (1986) - Autonomie du Campaniforme rhodano-rhénan : la question de la céramique domestique, in J.-P. Demoule et J. Guilaine dir., Le Néolithique de la France, Hommage à Gérard Bailloud, éd. Picard, Paris, p. 431-446.
GASCÓ J. (2004) - Les composantes de l’Âge du bronze, de la fin du Chalcolithique à l'Âge du bronze ancien en France méridionale, Cypsela, t. 15, p. 39-72.

GILLET F. (2000) - La phytosociologie synusiale intégrée. Guide méthodologique, Documents du Laboratoire d'écologie végétale, université de Neuchâtel, Institut de botanique, 68 p.

GONZALEZ MARCÉN P., MARTIN COLLIGA A., MORA TORCAL R. (1999) - Can Roqueta. Un establiment pagès prehistòric i medieval (Sabadell, Vallès Occidental), Excavacions Arqueològiques a Catalunya, t. 16, éd. DCGC, Barcelona, 447 p.

GUILAINE J. (1967) - La civilisation du vase campaniforme dans les Pyrénées françaises, éd. Gabelle, Carcassonne, 240 p.

GUILAINE J. (1972) - L'Âge du bronze en Languedoc occidental, Roussillon, Ariège, Mémoire de la Société préhistorique française, $\mathrm{n}^{\circ}$ 9, éd. Klincksiek, Paris, 460 p., 134 fig., 11 pl.

GUILAINE J. (1996) - Le Bronze ancien en Méditerranée occidentale, in C. Mordant et O. Gaiffe dir., Cultures et sociétés de l'Âge du bronze ancien en Europe, Actes du $117^{e}$ congrès national des Sociétés savantes, Clermont-Ferrand, 1992, éd. du CTHS, Paris, p. 3768.

GUILAINE J. et al. (1989) - Ornaisons, Médor. Archéologie et écologie d'un site de l'Âge du cuivre, de l'Âge du Bronze final et de l'Antiquité tardive, éd. CASR, Toulouse, $314 \mathrm{p}$.

GUILAINE J., CLAUSTRE F., LEMERCIER O., SABATIER P. (2002) Campaniformes et environnement culturel en France méditerranéenne, Bell Beakers Today, pottery, people, culture, symbols in third millennium Europe, International Colloquium, Riva del Garda (Italie), May 1998, éd. PAT, Trento, p. 229-275.

HÉNAFF X. (2003) - La céramique décorée du site artenacien de Ponthezières à Saint-Georges-d'Oléron (Charente-Maritime) dans son cadre régional, Bulletin de la Société préhistorique française, t. 100, $\mathrm{n}^{\circ} 4$, p. 733-755.

JACCARD P. (1908) - Nouvelles recherches sur la distribution florale, Bulletin de la Société vaudoise de Sciences naturelles, t. 44, p. 223270.

JOLIBERT B. (1988) - Le gisement campaniforme de Muret, éd. AEP, Toulouse, $137 \mathrm{p}$.

LEMERCIER O. (2004) - Le Campaniforme dans le Sud-Est de la France, Monographie d'Archéologie méditerranéenne, ${ }^{\circ}{ }^{\circ} 18$, éd. ADAL, Lattes, 518 p., 367 fig.

LOISON G. (2003) - L'Âge du bronze ancien en Auvergne, éd. AEP, Toulouse, 156 p., 106 fig.

PONS F., LAGARRIGUE A., avec la coll. de COLONGE D., JARRY M., MARTIN H. (2003) - Un habitat de plein air du Bronze ancien dans le Midi toulousain à Blagnac (Haute-Garonne) : le site de Cassagna 3, Documents d'Archéologie méridionale, $\mathrm{n}^{\circ} 26$, p. 7 41.

ROGER J.-M., FERRIER C., VALETTE P. (1988) - La structure campaniforme de Maupas (Calvisson, Gard) : note préliminaire, Bulletin de la Société d'études des sciences naturelles de Nîmes, p. 58 . 
ROUDIL J.-L. (1972) - L’Âge du bronze en Languedoc oriental, Mémoire de la Société préhistorique française, $\mathrm{n}^{\circ} 10$, éd. Klincksiek, Paris, 302 p., 109 fig., 27 pl.

ROUDIL J.-L., BAZILE F., SOULIER M. (1974) - L'habitat campaniforme de Saint-Côme-et-Maruéjols (Gard), Gallia Préhistoire, t. 17, $\mathrm{n}^{\circ} 1$, p. $181-213$

ROUSSOT-LARROQUE J. (1996) - Le Bronze ancien dans le Sud-Ouest de la France, in C. Mordant et O. Gaiffe dir., Cultures et sociétés de l'Âge du bronze ancien en Europe, Actes du $117^{e}$ congrès national des Sociétés savantes, Clermont-Ferrand, 1992, éd. du CTHS, Paris, p. 509-526.

SARTI L., MARTINI F. (1993) - Costruire la memoria. Archeologia preistorica a Sesto Fiorentino 1982-1992, éd. Garlatti e Razzai, Firenze, $89 \mathrm{p}$.

STRAHM C. (1998) - Il bicchiere campaniforme : fenomeno e cultura, in F. Nicolis et E. Mottes dir., Simbolo ed enigma : il bicchiere campaniforme e l'Italia nella preistoria europea del III millennio A.C., catalogo della mostra, Riva del Garda, 1998, Servizio Beni Culturali, Provincia Autonoma di Trento, Trento, p. 21-44.

TCHÉRÉMISSINOFF Y., MARSAC P., BERTHET A.-L., JARRY M., VIAROUGE M. (2005) - Essai de caractérisation d'un ensemble céramique campaniforme non décoré dans le Toulousain : le site de Cassagna 2, Bulletin de la Société préhistorique française, t. 102, $\mathrm{n}^{\circ} 2$, p. $381-400$.

THAUVIN-BOULESTIN E. (1998) - Le Bronze ancien et moyen des Grands Causses et des causses du Quercy, coll. Documents préhistoriques, ${ }^{\circ} 11$, éd. du CTHS, Paris, et Préhistoire du Sud-Ouest, Cressensac, 514 p., 164 fig.

VAQUER (1998) - Le Mourral, Trèbes (Aude). A fortified languedocian late neolithic site reoccupied by Bell Beakers, in M. Benz et S. Van Willigen dir., Some new approaches to the Bell Beakers "phenomenon": lost paradise...?, BAR international series, 690, éd. BAR, Oxford, p. 15-21.

VAQUER J. (1999) - Les origines préhistoriques de Carcassonne, Bulletin de la Société d'études scientifiques de l'Aude, t. XCIX, p. 1736.

VAQUER J., GANDELIN M., MARSAC R. (2004) - Le site du Roc d'En Gabit, Carcassonne (Aude), in H. Dartevelle coord., Auvergne et Midi, Actes des $V^{\text {s }}$ rencontres méridionales de Préhistoire récente, Clermont-Ferrand, 8-9 Novembre 2002, Préhistoire du Sud-Ouest, suppl. n 9, éd. PSO, Cressensac, p. 475-484.
VIGNAUD A. (2002) - L'éperon barré du Clos Marie-Louise, in Collectif, Archéologie du TGV Méditerranée. Fiches de synthèses. T. 2 : La Protohistoire, p. 573-576.

VILASECA ANGUERA S. (1973) - Reus y su entorno en la prehistoria, éd. AER, Reus, 2 vol., 282 p., 174 fig., 144 pl.

VITAL J. (1996) - État des travaux sur l'Âge du bronze ancien médiorhodanien, in C. Mordant et O. Gaiffe dir., Cultures et sociétés de l'Âge du bronze ancien en Europe, Actes du $117^{e}$ congrès national des Sociétés savantes, Clermont-Ferrand, 1992, éd. du CTHS, Paris, p. 325-338

VITAL J. (2004) - Du Néolithique final au Bronze moyen dans le SudEst de la France : 2200-1450 av. J.-C., Cypsela, t. 15, p. 11-38.

VITAL J., BROCHIER J.-L., DURAND J., PROST D., REYNIER P., RIMBAULT S. (1999a) - Roynac le Serre 1 (Drôme) : une nouvelle séquence holocène en Valdaine et ses occupations des Âges des métaux, Bulletin de la Société préhistorique française, t. 96, n 2, p. 225-240.

VITAL J., CONVERTINI F., JALLOT L., LEMERCIER O., LOISON G. dir. (1999b) - Projet collectif de recherche : Composantes culturelles des premières productions céramiques du Bronze ancien dans le SudEst de la France, rapport 1999 : Provence-Alpes-Côte d'Azur, Valence, 135 p., 33 fig.

VORUZ J. (1996) - Chronologie absolue de l'Âge du bronze ancien et moyen, in $\mathrm{C}$. Mordant et $\mathrm{O}$. Gaiffe dir., Cultures et sociétés de l'Âge du bronze ancien en Europe, Actes du $117^{e}$ congrès national des Sociétés savantes, Clermont-Ferrand, 1992, éd. du CTHS, Paris, p. 97-164.

Jean VAQUER

Directeur de recherche au CNRS

UMR 5608 TRACES-CRPPM-EHESS 39, allées Jules-Guesde, 31000 Toulouse vaquerjean@aol.com

Maxime REMICOURT Doctorant EHESS-CRPPM-UMR 5608 TRACES Dépôt de fouilles 5, rue de l'Olivier, 11000 Carcassonne m.remicourt@laposte.net 